\title{
Why myofascial release will never be evidence-based
}

\section{Robert F. Kidd}

\author{
Renfrew, Ontario, Canada
}

Myofascial release is an art form. Much depends on the innate talent and experience of the therapist. This is not to say that outcomes cannot be evaluated and compared with controls treated in some other way. However, in evaluating the outcome, the therapist cannot be excluded from the process. Even if all diagnostic variables could be controlled, any difference in outcome would have to be ascribed to the manual therapist using a particular technique, rather than to the technique itself.

Keywords: myofascial release - evidence base

A recent review ${ }^{1}$ in this journal of the evidence base for myofascial release produced slim pickings. All sorts of scientific reasons for why myofascial release might work were presented, but little evidence could be found that it does work. This should come as no surprise to anyone who actually uses this technique.

A number of reasons exist as to why this should be so, but before considering them let us look at the steps needed to prove any manual technique actually 'works'.

The overarching purpose of manual medicine is to provide relief from pain (or other symptom) of musculoskeletal origin. But to be able to do this, a series of clinical judgments must be made.

The first step is to make sure that the symptom is indeed of musculoskeletal origin. This is not always easy as posture and movement can be disturbed by non-musculoskeletal factors. The second step is to determine if the pain is of a kind that is likely to respond to manipulation (i.e. that it is due to somatic dysfunction). Neither of these steps is necessarily simple and the accuracy of both depends greatly on the skill and attentiveness of the physician. No instrument or measuring device can replace the manual skills of an experienced physician.

Correspondence to: Robert F. Kidd MD CM, 175 Argyle St S., Renfrew, Ontario K7V 1T6, Canada.

E-mail: drkidd@neuraltherapybook.com
After a diagnosis of somatic dysfunction has been made, the question of whether the somatic dysfunction is primary or secondary must then be answered; i.e. is it secondary to a viscero-somatic reflex, a somato-somatic reflex, a postural adaptation to a short leg, a cranial somatic dysfunction or other neuromusculoskeletal disturbance? Only if the somatic dysfunction is primary is manipulation likely to be of any lasting benefit.

When a primary somatic dysfunction is identified, a manipulative technique must be chosen (or designed). The choice depends on many attributes of both patient and physician, not least the physician's skills.

The manipulation must then be performed and the patient re-examined to evaluate its effect from a mechanical standpoint. If the patient's somatic dysfunction does not respond, the patient must be reevaluated to determine why.

Finally, the patient must be re-assessed at a certain interval afterward to evaluate the manipulation's success from the patient's point of view. Ultimately, this is the only measurement that matters!

Now, why is the effectiveness of manipulation so hard to prove? To begin with, the many variables in the above steps need to be controlled. Since many of these involve clinical judgement, it is essential that more than one physician be involved in all stages and different ones be responsible for evaluation and for treatment. All physicians would have to have similar skills to even hope 
to obtain some measure of inter-examiner reliability. This would be difficult but, in theory, not impossible.

Where difficult becomes impossible is when we consider the nature of the physician-patient interaction in both diagnosis and treatment. In manual medicine, by definition, the physician lays his hands upon the patient. The hands are the sensors that evaluate skin temperature, moisture and texture, the quality of the underlying subcutaneous tissue, muscles and fascia, and the position of bony landmarks. In addition, the hands are used to guide movement in assessment of quality and range of motion.

Skilled hands are sensitive instruments, but they also affect the tissues that they contact. Even gentle touch can elicit responses out of proportion to the force applied. This is true in both examination and treatment and is explained best by understanding that manipulation involves much more than simply transmitting mechanical forces.

The hands also transmit electromagnetic signals that have biological effects. This has been studied most in the hands of 'healers' but there is no reason to believe that this does not apply to many, if not most, manual therapists. ${ }^{2}$ Some osteopathic techniques make explicit use of this energy to treat spinal and other somatic dysfunction. ${ }^{3}$

Not only do the hands transmit signals but the very presence of another person alters biological rhythms such as brain waves and heart rate. ${ }^{4}$ The EEGs of 'healers' when in a healing state consistently average about $7.8-8.0 \mathrm{~Hz},{ }^{5}$ a range that corresponds with the specific frequencies that facilitate healing in various tissues ( $7 \mathrm{~Hz}$ for bone, $10 \mathrm{~Hz}$ for ligaments ${ }^{6}$ ).

Many other examples could be given of the subtle interactions of manual therapist and physician during the therapeutic encounter. ${ }^{7,8}$ The point to be made here is that innumerable, uncontrollable variables in manual medicine make researching the efficacy of manipulation extremely difficult.

This becomes even more evident when studying the technique of myofascial release. Before myofascial release is attempted, all the preliminary steps that precede any form of manipulation must be taken (as described above). Then the myofascia must be 'engaged' with just the right force and at just the right angle. This is a process of 'taking up the slack' to the point that a very slight resistance is felt. Once the tissues are engaged, a manual force (usually traction) is applied in such a way that one feels the myofascia release. Not all the tissues release at the same time. In practice, tissues release in unpredictable ways, and the therapist must be constantly sensing the release and adjusting the amount and direction of the force applied.

The cues that the patient sends to the therapist can be extremely subtle. In fact, a skilled therapist picks up signals transcending the sense of touch. He or she senses 'through the mind's eye' where the tissues are releasing and adjusts the direction and angle of force accordingly. This process becomes a 'dance' with a constant two-way flow of information.

What the above description suggests is that myofascial release is an art form. Much depends on the innate talent and experience of the therapist. This is not to say that outcomes cannot be evaluated and compared with controls treated in some other way. However, in evaluating the outcome, the therapist cannot be excluded from the process. Even if all diagnostic variables could be controlled, any difference in outcome would have to be ascribed to the manual therapist using a particular technique, rather than to the technique itself.

\section{References}

1. Remvig L, Ellis RM, Patjin J. Myofascial release: an evidence-based treatment approach? Int Musculoskel Med 2008; 30: 29-35.

2. Seto A, Kusaka C, Nakazato S et al. Detection of extraordinary large biomagnetic field strength from human hand. Acupuncture Electrother Res Int J 1992; 17: 75-94.

3. Upledger JE, Vredevoogd JD. Craniosacral therapy. Chicago, IL: Eastland Press, 1983; 139-140.

4. Russek LG, Schwartz GE. Interpersonal heart-brain registration and the perception of parental love: a 42-year follow-up of the Harvard Mastery of Stress Study. Subtle Energies 1994; 5: 195-208.

5. Beck R. Mood modification with ELF magnetic fields: a preliminary exploration. Archaeus 1986; 4: 48.

6. Sisken BF, Walker J. Therapeutic aspects of electromagnetic fields for soft-tissue healing. In: Blank M. (ed) Electromagnetic fields: biological interactions and mechanisms. Advances in Chemistry Series 250. Washington, DC: American Chemical Society, 1995; 227-285.

7. Oschman JL. Energy medicine: The scientific basis. Edinburgh: Churchill Livingstone, 2000.

8. Oschman JL. Energy medicine in therapeutics and human performance. Edinburgh: Butterworth Heineman, 2003. 